\title{
ANÁLISE DE TRILHA E VARIAÇÃO GENÉTICA NA CULTURA DO MILHO EM CONDIÇÕES DE CERRADO
}

\author{
Edmar Vinicius de Carvalho ${ }^{1}$, Flávio Sérgio Afférri ${ }^{2}$, Joênes Mucci Peluzio ${ }^{1}$, Gustavo André Colombo ${ }^{3}$, Michel \\ Antônio Dotto ${ }^{4}$ \\ ${ }^{1}$ Universidade Federal do Tocantins - UFT, TO. ${ }^{2}$ Universidade Federal de São Carlos - UFSCAR, SP. ${ }^{3}$ Instituto Federal do \\ Amazonas - IFAM, AM. ${ }^{4}$ GDM Seeds. E-mail: carvalho.ev@uft.edu.br
}

\section{RESUMO}

O melhoramento genético pode propiciar ganhos em produtividade, com o desenvolvimento de genótipos, explorando a variabilidade genética existente na cultura do milho. Outro fator, que aumenta a eficiência nos programas de melhoramento, é o conhecimento da relação de componentes produtivos e de eficiência com a produtividade de grãos. O objetivo deste trabalho foi avaliar a variação genotípica de componentes produtivos, índices de eficiência na cultura do milho e sua relação com a produtividade de grãos. Entre as safras de 2009/2010 e 2013/2014, foram conduzidos 24 experimentos de milho sob delineamento de blocos ao acaso. Os experimentos se diferiram quanto a quantidade e tipo de genótipos, tipo e dose de adubação de semeadura e de cobertura nitrogenada. Dentro de cada safra, as médias dos experimentos foram submetidas a análises estatísticas para a classificação dos mesmos em favoráveis e desfavoráveis para a expressão da produtividade de grãos. Assim, as análises de trilha, tendo a produtividade como atributo dependente e os demais como explicativos e; a estimativa dos coeficientes de variação experimental e genotípico, foram realizadas separadamente para experimentos favoráveis e desfavoráveis, em cada safra. As estimativas da variabilidade genética e da associação dos atributos com a produtividade de grãos sofreram influência com mudança das condições de cultivo (favorável; desfavorável). Foi possível verificar que os índices estimados, potencial produtivo e eficiência produtiva, explicaram melhor a variação da produtividade de grãos que o número de grãos e massa média do grão.

Palavras-chave: associação de caracteres; melhoramento; nitrogênio; Tocantins; Zea mays L.

\section{PATH ANALYSIS AND GENETIC VARIATION ON MAIZE UNDER CERRADO CONDITIONS}

\begin{abstract}
The plant breeding can be promoting the grain yield increase with development of new genotypes and the exploration the genetic variability that there was in maize. The other factor for the elevate plant breeding efficiency is the knowledge about the relationship among productive and efficiency components with the grain yield. The aim of this work was to evaluate the genotypic variation of productive components and efficiency index in the maize and the relationship these traits with the grain yield. Among the 2009/2010 and 2013/2014 seasons, they were conducted 24 maize experiments under randomized block design. The experiments were different in function of seeding date, type and quantity of genotypes, seeding and covered fertilization. For each season, the experiments mean were submitted the statistical analysis for the distinction of the favorable and unfavorable conditions for the maize grain yield. In each condition, they were done path analysis with the grain yield was the principal trait and the others were the explicative traits and; they were estimate the experimental and genotypic variation coefficients, yet. The estimates of genetic variation and association of the traits with the grain yield had shown influence with change of the growth conditions (favorable; unfavorable). It was possible the verify that the two-index estimated (productive potential and efficiency) were better to explain the grain yield variation than the potential grain number and men grain weight.
\end{abstract}

Keywords: association; nitrogen; plant breeding; Tocantins; Zea mays L. 


\section{INTRODUÇÃO}

O aumento da produção, na cultura do milho, pode ser alcançado com o cultivo em épocas não tradicionais, em novas fronteiras agrícolas e/ou com o uso de genótipos mais eficientes (CAIXETA et al., 2015). Por meio do melhoramento genético, pode-se desenvolver genótipos que sejam adaptados a diversas condições de cultivo (BU et al., 2015) e que podem representar ganhos de 118,5 a $173,0 \mathrm{~kg} \mathrm{ha}^{-1}$ por ano de lançamento do material (ECHARTE et al., 2000; CHEN et al., 2013).

A cultura do milho, em comparação a plantas C3, apresenta maior variação na produção em função da quantidade de água disponível (ZWART; BASTIAANSSEN, 2004), bem como da alta variação fenotípica intraespecífica (BAUTE et al., 2015). No entanto, segundo $\mathrm{Wu}$ et al. (2011), a variabilidade genética em genótipos de milho, ainda, é pouco conhecida, o que torna fundamental a realização de pesquisas constantes nas diversas condições de cultivo (DOVALE et al., 2013).

A produtividade de grãos, nos cereais em geral, é resultado da interação entre diversos atributos, como a quantidade de grãos produzidos e a massa de cada um (ORDÓNEZ et al., 2015), os quais são utilizados na determinação do potencial produtivo da planta (CHEN et al., 2013) e suscetíveis a variação ambiental (CHEN et al., 2015). O número de grãos é afetado tanto por condições anteriores ao florescimento quanto no período de enchimento de grãos (ORDÓNEZ et al., 2015), enquanto que e a massa dos grãos está relacionada ao crescimento dos mesmos que por sua vez é limitado pela disponibilidade de assimilados do florescimento à maturação fisiológica (BORRÁS et al., 2004).

A utilização dos valores da produtividade de grãos por planta é observada em trabalhos recentes na cultura do milho (MUHAMMAD et al., 2015; ORDÓNEZ et al., 2015; TOKATLIDIS et al., 2015), e que se justifica pela necessidade de aumentar o potencial produtivo da planta (MAPHUMULO et al., 2015), principalmente, quando o objetivo é o desenvolvimento de genótipos adaptados a ambientes heterogêneos (TOKATLIDIS et al., 2015) e adversos ao crescimento/desenvolvimento vegetal.

O conhecimento da relação da produtividade de grãos com seus componentes é considerado importante para aumentar a eficiência num programa de melhoramento genético (FILIPOVIC et al., 2014; MAPHUMULO et al., 2015; CABRAL et al., 2016). No entanto, essa relação pode ser alterada por condições ambientais (CARPICI; CELIK, 2010; FILIPOVIC et al., 2014), como os estresses (SULTAN et al., 2014) e assim, é importante a realização destes estudos em novas fronteiras agrícolas, como o cerrado brasileiro.

Neste ponto, o coeficiente de correlação linear de Pearson representa a associação linear entre dois atributos sem revelar as relações de causa-efeito (PEREIRA et al., 2013; CABRAL et al., 2016), que podem ser estudadas a partir da análise de trilha por meio dos efeitos diretos e indiretos (CARPICI; CELIK, 2010). De acordo com O'Neill et al. (2006), atributo ideal para realização de seleção indireta será aquele que apresentar correlação $(r>$ $|0,90|)$ com o atributo alvo, possuir alta herdabilidade com variabilidade genética e que seja de fácil mensuração e estável durante sua avaliação.

A hipótese do trabalho é de que a condição de cultivo exerce influência na associação dos atributos e na manifestação variabilidade genética dos mesmos. Assim, o objetivo do presente trabalho foi avaliar a variação genotípica de componentes produtivos e índices de eficiência na cultura do milho e a relação dos mesmos com a produtividade de grãos.

\section{MATERIAL E MÉTODOS}

Ao todo foram conduzidos 24 experimentos de campo, com diferentes genótipos de milho, no Estado do Tocantins (municípios de Gurupi - 280 m, 114' S, 4903' O; e Palmas - 220 m, 1010' S, 4821' O), durante os anos de 2009 e 2014 e com uso do delineamento de blocos ao acaso em Latossolo Vermelho Amarelo textura franco-argilo-arenosa.

O clima da região é do tipo tropical, com períodos de chuva intensa no verão e estiagem total no inverno, segundo a classificação de Köppen, com temperatura média de $28^{\circ} \mathrm{C}$, mínimas e máximas e de 20,7 e 32,60 C e precipitação média de $1.684 \mathrm{~mm}$ ao ano. A descrição geral dos experimentos está presente na Tabela 1 e os resultados médios das análises químicas de solo, em cada município e ano/safra, na Tabela 2. 
Tabela 1. Descrição geral dos experimentos de campo na avaliação de genótipos de milho

\begin{tabular}{|c|c|c|c|c|c|c|c|}
\hline \multirow{2}{*}{ Exp. } & \multirow{2}{*}{ Ano/safra } & \multirow{2}{*}{ Data semeadura } & \multicolumn{2}{|r|}{ Genótipos } & \multirow{2}{*}{ Repetições } & \multicolumn{2}{|c|}{ Adubação (kg ha $\left.{ }^{-1}\right)$} \\
\hline & & & Qtde & Tipo & & Semeadura ${ }^{*}$ & Cobertura $^{* *}$ \\
\hline 1 & $09 / 10-1$ & 14.12 .09 & 32 & $\mathrm{HS} ; \mathrm{HT}$ & 2 & 600 & ON \\
\hline 2 & $09 / 10-1$ & 14.12.09 & 32 & HS; HT & 2 & 600 & $144 N$ \\
\hline 3 & 09/10 - II & 21.11 .09 & 21 & HS; HT & 2 & 600 & ON \\
\hline 4 & $09 / 10-11$ & 21.11 .09 & 21 & $\mathrm{HS} ; \mathrm{HT}$ & 2 & 600 & $144 N$ \\
\hline 5 & $09 / 10$ - II & $03.12 .09^{* * *}$ & 21 & $\mathrm{HS} ; \mathrm{HT}$ & 2 & 600 & ON \\
\hline 6 & 09/10 - II & $03.12 .09^{* * *}$ & 21 & $\mathrm{HS} ; \mathrm{HT}$ & 2 & 600 & $144 N$ \\
\hline 7 & 09/10 - II & 14.12 .09 & 21 & $\mathrm{HS} ; \mathrm{HT}$ & 2 & 600 & ON \\
\hline 8 & 09/10 - II & 14.12.09 & 21 & $\mathrm{HS} ; \mathrm{HT}$ & 2 & 600 & $144 N$ \\
\hline 9 & 10 & 06.06 .10 & 29 & HS; HT; PPA`s & 2 & 500 & ON \\
\hline 10 & 10 & 06.06 .10 & 29 & HS; HT; PPA`s & 2 & 500 & $125 \mathrm{~N}$ \\
\hline 11 & 10 & 06.06 .10 & 29 & HS; HT; PPA`s & 2 & 40.000 & ON \\
\hline 12 & 10 & 06.06 .10 & 29 & HS; HT; PPA`s & 2 & 40.000 & $125 \mathrm{~N}$ \\
\hline 13 & $12 / 13$ & 30.10 .12 & 30 & PPA's; L & 3 & 500 & 500 \\
\hline 14 & $12 / 13$ & 30.10 .12 & 30 & PPA's; L & 3 & 500 & $150 \mathrm{~N}$ \\
\hline 15 & $12 / 13$ & 06.03 .13 & 30 & PPA's; L & 3 & 500 & 500 \\
\hline 16 & $12 / 13$ & 06.03 .13 & 30 & PPA's; L & 3 & 500 & $150 \mathrm{~N}$ \\
\hline 17 & $13 / 14-I$ & 15.01 .14 & 12 & PPA's, V, HD & 3 & 500 & $20 \mathrm{~N}$ \\
\hline 18 & $13 / 14-I$ & 15.01 .14 & 12 & PPA's, V, HD & 3 & 500 & $80 \mathrm{~N}$ \\
\hline 19 & $13 / 14-I$ & 15.01 .14 & 12 & PPA's, V, HD & 3 & 500 & $140 \mathrm{~N}$ \\
\hline 20 & $13 / 14-I$ & 15.01.14 & 12 & PPA's, V, HD & 3 & 500 & $200 \mathrm{~N}$ \\
\hline 21 & $13 / 14$ - II & 25.02 .14 & 12 & PPA's, V, HD & 3 & 500 & $20 \mathrm{~N}$ \\
\hline 22 & $13 / 14$ - II & 25.02 .14 & 12 & PPA's, V, HD & 3 & 500 & $80 \mathrm{~N}$ \\
\hline 23 & $13 / 14-I I$ & 25.02 .14 & 12 & PPA's, V, HD & 3 & 500 & $140 \mathrm{~N}$ \\
\hline 24 & $13 / 14$ - II & 25.02 .14 & 12 & PPA's, V, HD & 3 & 500 & $200 N$ \\
\hline
\end{tabular}


Tabela 2. Análises químicas do solo, na camada de 0 a $20 \mathrm{~cm}$, em Gurupi e Palmas, Tocantins

\begin{tabular}{cccccccc}
\hline $\begin{array}{c}\text { Ano/ } \\
\text { safra }\end{array}$ & $\begin{array}{c}\mathrm{P} \\
\left(\mathrm{mg} \mathrm{dm}^{-3}\right)\end{array}$ & $\begin{array}{c}\mathrm{K} \\
\left(\mathrm{cmol} \mathrm{dm}^{-3}\right)\end{array}$ & $\begin{array}{c}\mathrm{Ca}+\mathrm{Mg} \\
\left(\mathrm{cmol} \mathrm{dm}^{-3}\right)\end{array}$ & $\begin{array}{c}\mathrm{Al}+\mathrm{H} \\
\left(\mathrm{cmol} \mathrm{dm}^{-3}\right)\end{array}$ & $\begin{array}{c}\mathrm{pH} \\
\left(\mathrm{CaCl}_{2}\right)\end{array}$ & $\begin{array}{c}\mathrm{MO} \\
\left(\mathrm{g} \mathrm{dm}^{-3}\right)\end{array}$ \\
\hline $09 / 10-\mathrm{I}$ & 7,40 & 0,18 & 4,00 & 3,80 & $6,000^{* *}$ & 52,40 & 13,00 \\
$09 / 10-\mathrm{II}^{*}$ & 8,40 & 0,15 & 3,50 & 3,70 & $6,00^{* *}$ & 49,60 & 11,00 \\
10 & 4,80 & 0,10 & 2,50 & 3,40 & $5,20^{* *}$ & 42,20 & 4,00 \\
$12 / 13$ & 10,40 & 0,13 & 2,30 & 2,30 & 5,20 & 51,60 & 20,00 \\
$13 / 14-\mathrm{I}^{1}$ & 1,80 & 0,04 & 1,10 & 2,40 & 4,70 & 32,40 & 16,00 \\
$13 / 14-\mathrm{II}^{2}$ & 3,70 & 0,12 & 2,20 & 2,30 & 5,10 & 50,40 & 15,00 \\
\hline
\end{tabular}

Município de Palmas-TO, todos os demais resultados referentes ao município de Gurupi-TO; ${ }^{* *} \mathrm{pH}^{\text {em água; }}{ }^{1}$ Semeadura em janeiro; ${ }^{2}$ Semeadura em fevereiro.

A densidade de plantio, nos experimentos, foi ajustada a 40.000 a 62.500 plantas ha ${ }^{-1}$ após realização de desbaste, utilizando espaçamento entre linhas variando de 0,75 a 1,0 m, conforme os trabalhos realizados por Carvalho et al. (2011, 2012, 2014, 2016, 2017) e que relatam a origem dos genótipos utilizados. As demais práticas culturais, seguiram as recomendações técnicas de manejo da cultura para a região dos cerrados.

Após a maturação fisiológica, as espigas de plantas representativas das parcelas (mínimo de 10 plantas) foram colhidas e despalhadas para determinação dos seguintes atributos: número potencial de grãos (NPG), pela multiplicação do número de grãos por fileira na espiga e o número de fileiras de grãos; produtividade de grãos (PG) em g planta $^{-1}$, a $13 \%$ de umidade; massa média do grão (M1G), em g a 13\% de umidade, obtida pela divisão da massa de 100 grãos por 100; potencial produtivo (PP), obtido pela multiplicação entre NPG e M1G; eficiência produtiva (EP), obtida pela relação entre a PG e o PP.

Na relação acima, o numerador representa a produtividade de grãos por planta, em gramas, que foi aferida, ou seja, o valor real por planta, enquanto que, o denominador representa 0 potencial produtivo da produtividade de grãos por planta, pois é obtido pela multiplicação entre o número potencial de grãos por espiga e a massa média de cada grão. Assim, resume a relação entre o potencial produtivo e o nível de produção atingido por determinado genótipo na condição de cultivo que foi submetido.

Após a tabulação dos dados de todos os experimentos, foi verificada a normalidade dos erros não sendo necessária a realização da transformação dos dados.

\section{Análises prévias}

Os dados dos experimentos foram submetidos a análise de variância conjunta dentro de cada safra, considerando os genótipos como tratamentos e com efeito fixo e a combinação dos diferentes fatores de condição de cultivo como ambiente e com efeito aleatório. Tais análises foram feitas para separar os experimentos em favoráveis ou desfavoráveis para expressão da produtividade de grãos, por meio do teste de médias Scott-Knott $(p<0,05)$, em cada safra. Desta forma, os experimentos favoráveis foram aqueles classificados no grupo de médias estatisticamente superior e; os desfavoráveis, os classificados no grupo de médias estatisticamente inferior.

\section{Análises finais}

Em cada um dos experimentos (favoráveis; desfavoráveis) e em cada safra, foi realizada análise de variância para a estimação do coeficiente de variação experimental e genotípico e do valor de probabilidade do teste $F$ para a fonte de variação Genótipos, nos seguintes atributos: NPG, M1G, PP e EP.

Ainda, de forma separada para o conjunto de dados dos experimentos favoráveis e desfavoráveis, a análise de correlação linear foi realizada entre os atributos mensurados para a geração da matriz utilizada na análise de trilha. Nesta análise, a produtividade de grãos foi considerada como atributo principal e os demais como explicativos e, que foi realizada sob efeito de colinearidade com uso da constante $\mathrm{K}$ (valores de 0,04 a 0,06 ). Este procedimento foi realizado com a intenção do número de condições (NC) ser menor que 100 e o do fator de inflação da variância (FIV) ser menor que 10 em cada atributo explicativo (PEREIRA et al., 2013). Todas as análises foram realizadas empregando o software $R(R$ CORE TEAM, 2015).

\section{RESULTADOS E DISCUSSÃO Análises prévias}

Por meio do teste de médias Scott-Knott ( $p$ $<0,05)$, a classificação dos experimentos em favoráveis e desfavoráveis, em cada ano/safra, está apresentada na Tabela 3. De acordo com estes resultados, pode-se observar que a dose de 
nitrogênio em cobertura foi o principal fator para que determinado experimento fosse considerado como favorável, por proporcionar média estatisticamente superior a produtividade de grãos, em comparação aos demais, dentro de cada ano/safra.

Tabela 3. Identificação dos experimentos em favoráveis (médias estatisticamente superiores) e desfavoráveis (médias estatisticamente inferiores), de acordo com a intepretação do resultado do teste Scott-Knott ( $p$ $<0,05)$, em cada ano/safra

\begin{tabular}{|c|c|c|}
\hline Ano/Safra & Desfavorável & Favorável \\
\hline Safra 09/10 - I & $\begin{array}{c}0 \mathrm{~N} \\
\left(100,0 \mathrm{~g} \mathrm{planta}^{-1}\right)\end{array}$ & $\begin{array}{c}144 \mathrm{~N} \\
\left(113,5 \text { g planta }^{-1}\right) \\
\end{array}$ \\
\hline Safra 09/10 - II & $\begin{array}{c}0 \mathrm{~N} \\
\left(102,8 \mathrm{~g} \mathrm{planta}^{-1}\right)\end{array}$ & $\begin{array}{c}144 \text { N } \\
\left(124,7 \text { g planta }^{-1}\right)\end{array}$ \\
\hline Entressafra 10 & $\begin{array}{c}0 \mathrm{~N}^{1} \text { e } 125 \mathrm{~N}^{2} \\
\left(103,6 \text { a } 109,6 \text { g planta }^{-1}\right)\end{array}$ & $\begin{array}{c}125 \mathrm{~N}^{3} \\
\left(131,1 \text { g planta }^{-1}\right)\end{array}$ \\
\hline Safra $12 / 13$ & $\begin{array}{c}0 \mathrm{~N}^{4} \\
\left(55,3 \mathrm{~g} \mathrm{planta}^{-1}\right)\end{array}$ & $\begin{array}{c}0 \text { N e } 150 \text { N }^{5} \\
\left(85,7 ; 87,7 \text { g planta }^{-1}\right)\end{array}$ \\
\hline Safra $13 / 14$ - I & $\begin{array}{c}20 \mathrm{~N} \text { e } 140 \mathrm{~N} \\
\left(54,8 ; 55,6 \text { g planta }^{-1}\right)\end{array}$ & $\begin{array}{c}200 \mathrm{~N} \text { e } 80 \mathrm{~N} \\
\left(60,3 ; 61,0 \text { g planta }^{-1}\right)\end{array}$ \\
\hline Safra $13 / 14$ - II & $\begin{array}{c}20 \mathrm{~N} \\
\left(80,4 \mathrm{~g} \mathrm{planta}^{-1}\right)\end{array}$ & $\begin{array}{c}140 \mathrm{~N}, 200 \mathrm{~N} \text { e } 80 \mathrm{~N} \\
\left(88,2 \text { a } 90,7 \text { g planta }^{-1}\right)\end{array}$ \\
\hline
\end{tabular}

${ }^{1}=$ referente a dose de $0 \mathrm{~kg} \mathrm{ha}^{-1}$ de $\mathrm{N}$ em cobertura, tanto com adubação orgânica ou mineral de semeadura; ${ }^{2}=$ referente a dose de $125 \mathrm{~kg} \mathrm{ha}^{-1}$ de N em cobertura com adubação mineral de semeadura; ${ }^{3}=$ referente a dose de $125 \mathrm{~kg}^{2}$ $\mathrm{ha}^{-1}$ de $\mathrm{N}$ em cobertura com adubação orgânica de semeadura; ${ }^{4}=$ referente a dose $0 \mathrm{~kg} \mathrm{ha}^{-1} \mathrm{de} \mathrm{N} \mathrm{em} \mathrm{cobertura} \mathrm{quando}$ semeadura em março; ${ }^{5}=$ referente as doses 0 e $150 \mathrm{~kg} \mathrm{ha}^{-1} \mathrm{em}$ cobertura quando semeadura em outubro.

\section{Análises finais}

No número potencial de grãos (NPG) e na massa média do grão (M1G), diferença significativa entre os genótipos avaliados foi detectada em $10 \mathrm{e}$ 9 das 12 análises, respectivamente (Tabelas 4 e 5). No potencial produtivo (PP), mesmo resultado foi observado em 8 das 12 análises, e por fim, na eficiência produtiva (EP) em 3 de 12 . Estas diferenças podem ser explicadas em função do atributo que foi mensurado/estimado, bem como dos genótipos e das condições ambientais na avaliação (MAPHUMULO et al., 2015).

A variação experimental, medida pelo coeficiente de variação experimental (CV), foi superior a variação genotípica $(\mathrm{CVg})$ em todas as condições ambientais e genótipos avaliados para a EP e em, 10 de 12 das situações possíveis para o PP (Tabelas 4 e 5). Makumbi et al. (2015) observaram, em diversos atributos avaliados na cultura do milho, que a variância genética foi inferior a variância experimental, independente da condição ambiental de avaliação. Segundo Sserumaga et al. (2016), tais resultados possuem relação com o fato de que o ambiente exerce maior efeito na explicação da variância na maioria dos atributos.

Num programa de melhoramento, o ideal é que essa relação (CV/CVg) seja inferior a unidade, pois o progresso/sucesso está intrinsicamente relacionado com a variabilidade genética (MAPHUMULO et al., 2015). Isto demonstra a importância do conhecimento da variância dos atributos (MUHAMMAD et al., 2015) e do efeito do ambiente na manifestação da mesma. 
Tabela 4. Coeficiente de variação experimental e genotípico $\left(C V ; C V_{g}\right)$, valor $P$ do teste $F$ para genótipos ( $p-$ Teste $F_{G}$ ) de quatro atributos e suas respectivas correlações $(r)$ e efeitos diretos sob a produtividade de grãos na avaliação de diferentes genótipos de milho, em experimentos desfavoráveis ${ }^{1}$ em cada safra, Tocantins

\begin{tabular}{cccccc}
\hline \multicolumn{7}{c}{ Safra 2009/10 $(\mathrm{n}=64)$} \\
\hline Atributo & $\mathrm{CV}$ & $\mathrm{CV}_{\mathrm{g}}$ & $\mathrm{p}$ - Teste $\mathrm{F}_{\mathrm{G}}$ & $\mathrm{r}$ & Efeito direto \\
\hline M1G & 8,83 & 10,02 & 0,00 & $0,53^{* *}$ & 0,25 \\
NPG & 22,92 & - & 0,90 & $0,55^{* *}$ & 0,37 \\
PP & 22,56 & 5,42 & 0,38 & $0,76^{* *}$ & 0,44 \\
EP & 17,09 & 5,52 & 0,30 & $0,43^{* *}$ & 0,62 \\
\hline \multicolumn{7}{c}{$\mathrm{R}^{2} /$ Efeito variável residual } \\
\hline \multicolumn{7}{c}{}
\end{tabular}

\begin{tabular}{cccccc}
\multicolumn{7}{c}{ Safra 2009/10 $(\mathrm{n}=126)$} \\
\hline M1G & 8,76 & 3,82 & 0,01 & $0,27^{* *}$ & 0,18 \\
NPG & 14,42 & 8,97 & 0,00 & $0,39^{* *}$ & 0,26 \\
PP & 16,95 & 5,49 & 0,12 & $0,51^{* *}$ & 0,38 \\
EP & 29,03 & 4,44 & 0,88 & $0,71^{* *}$ & 0,82 \\
\hline \multicolumn{7}{c}{$\mathrm{R}^{2} /$ Efeito variável residual } \\
\hline \multicolumn{7}{c}{$0,92 / 0,28$} \\
\hline M1G & 11,59 & 7,65 & 0,25 \\
NPG & 18,42 & 12,36 & 0,00 & 0,28 \\
PP & 21,89 & 10,16 & 0,00 & 0,14 & 0,23 \\
EP & 55,65 & 11,64 & 0,00 & $0,30^{* *}$ & 0,62 \\
\hline \multicolumn{7}{c}{$\mathrm{R}^{2} /$ Efeito variável residual } \\
\hline \multicolumn{7}{c}{0,21} & $0,39^{* *}$ & $0,43 / 0,75$ \\
\hline
\end{tabular}

Safra $2012 / 13(n=90)$

\begin{tabular}{cccccc}
\hline M1G & 12,51 & 12,34 & 0,00 & $0,38^{* *}$ & 0,16 \\
NPG & 25,75 & 18,19 & 0,00 & $0,77^{* *}$ & 0,31 \\
PP & 27,67 & 20,40 & 0,00 & $0,86^{* *}$ & 0,42 \\
EP & 25,54 & - & 0,75 & $0,59^{* *}$ & 0,45 \\
\hline \multicolumn{7}{c}{$\mathrm{R}^{2} /$ Efeito variável residual } \\
\hline
\end{tabular}

Safra $2013 / 14(n=72)$

\begin{tabular}{cccccc}
\hline M1G & 13,57 & 2,11 & 0,12 & $0,38^{* *}$ & 0,34 \\
NPG & 8,40 & 5,66 & 0,00 & $0,48^{* *}$ & 0,22 \\
PP & 15,25 & 5,90 & 0,01 & $0,59^{* *}$ & 0,43 \\
EP & 20,12 & 5,05 & 0,37 & $0,56^{* *}$ & 0,81 \\
\hline \multicolumn{7}{c}{$\mathrm{R}^{2} /$ Efeito variável residual } & $0,94 / 0,25$ \\
\hline
\end{tabular}

Safra $2013 / 14(n=36)$

\begin{tabular}{cccccc}
\hline M1G & 11,06 & 6,94 & 0,06 & $0,36^{*}$ & 0,19 \\
NPG & 10,19 & 10,25 & 0,00 & $0,49^{* *}$ & 0,19 \\
PP & 13,83 & 12,37 & 0,01 & $0,65^{* *}$ & 0,48 \\
EP & 14,46 & 5,74 & 0,21 & $0,65^{* *}$ & 0,72 \\
\hline \multicolumn{7}{c}{$\mathrm{R}^{2} /$ Efeito variável residual }
\end{tabular}

${ }^{1}=$ classificados de acordo com o resultado do teste de médias Scott-Knott $(p<0,05)$, vide Tabela 3. M1G = massa média do grão; NPG = número potencial de grãos; $P P=$ potencial produtivo; $E P=$ eficiência produtiva; $R^{2}=$ coeficiente de determinação; * $\mathrm{e}^{* *}=$ significativo pelo teste $\mathrm{t}(p<0,05 ; p<0,01$, respectivamente).

Tabela 5. Coeficiente de variação experimental e genotípico $\left(C V ; \mathrm{CV}_{\mathrm{g}}\right)$, valor $\mathrm{P}$ do teste $\mathrm{F}$ para genótipos $(\mathrm{p}-$ Teste $F_{G}$ ) de quatro atributos e suas respectivas correlações $(r)$ e efeitos diretos sob a produtividade de grãos na avaliação de diferentes genótipos de milho, em experimentos favoráveis ${ }^{1}$ em cada safra, Tocantins

Safra 2009/10 ( $n=64)$

$\begin{array}{llllll}\text { Atributo } & \mathrm{CV} & \mathrm{CV}_{\mathrm{g}} & \mathrm{p} \text { - Teste } \mathrm{F}_{\mathrm{G}} & \mathrm{r} & \text { Efeito direto }\end{array}$




\begin{tabular}{|c|c|c|c|c|c|}
\hline M1G & 9,1 & 6,95 & 0,02 & 0,14 & 0,24 \\
\hline NPG & 16,01 & 9,26 & 0,08 & $0,54^{* *}$ & 0,37 \\
\hline $\mathrm{PP}$ & 17,38 & 8,85 & 0,13 & $0,59^{* *}$ & 0,43 \\
\hline EP & 15,98 & 6,99 & 0,19 & $0,56^{* *}$ & 0,78 \\
\hline \multicolumn{5}{|c|}{$\mathrm{R}^{2} /$ Efeito variável residual } & $0,92 / 0,28$ \\
\hline \multicolumn{6}{|c|}{ Safra $2009 / 10(n=126)$} \\
\hline M1G & 7,41 & 4,35 & 0,00 & 0,15 & 0,24 \\
\hline NPG & 17,9 & 7,98 & 0,03 & $0,26^{* *}$ & 0,23 \\
\hline PP & 16,37 & 9,34 & 0,00 & $0,35^{* *}$ & 0,43 \\
\hline EP & 18,99 & 2,27 & 0,12 & $0,65^{* *}$ & 0,92 \\
\hline \multicolumn{5}{|c|}{$\mathrm{R}^{2}$ / Efeito variável residual } & $0,84 / 0,40$ \\
\hline \multicolumn{6}{|c|}{ Entresafra $2010(n=58)$} \\
\hline M1G & 8,72 & 7,08 & 0,01 & $0,39^{* *}$ & 0,14 \\
\hline NPG & 12,66 & 16,62 & 0,00 & $-0,14$ & 0,16 \\
\hline $\mathrm{PP}$ & 13,31 & 12,43 & 0,00 & 0,08 & 0,41 \\
\hline EP & 25,60 & 24,64 & 0,00 & $0,81^{* *}$ & 0,99 \\
\hline \multicolumn{5}{|c|}{$\mathrm{R}^{2}$ / Efeito variável residual } & $0,87 / 0,36$ \\
\hline \multicolumn{6}{|c|}{ Safra $2012 / 13(n=180)$} \\
\hline M1G & 8,97 & 9,34 & 0,00 & $0,38^{* *}$ & 0,19 \\
\hline NPG & 16,01 & 16,23 & 0,00 & $0,61^{* *}$ & 0,33 \\
\hline PP & 17,72 & 18,00 & 0,00 & $0,74^{* *}$ & 0,38 \\
\hline EP & 22,15 & 9,58 & 0,01 & $0,60^{* *}$ & 0,61 \\
\hline \multicolumn{5}{|c|}{$\mathrm{R}^{2}$ / Efeito variável residual } & $0,92 / 0,28$ \\
\hline \multicolumn{6}{|c|}{ Safra $2013 / 14(n=72)$} \\
\hline M1G & 16,31 & - & 0,78 & $0,39^{* *}$ & 0,29 \\
\hline NPG & 9,10 & 6,81 & 0,00 & $0,48^{* *}$ & 0,23 \\
\hline $\mathrm{PP}$ & 19,05 & 4,47 & 0,07 & $0,57^{* *}$ & 0,41 \\
\hline EP & 21,97 & 4,13 & 0,28 & $0,59^{* *}$ & 0,79 \\
\hline \multicolumn{5}{|c|}{$\mathrm{R}^{2}$ / Efeito variável residual } & $0,93 / 0,27$ \\
\hline \multicolumn{6}{|c|}{ Safra $2013 / 14(n=108)$} \\
\hline M1G & 11,66 & 7,26 & 0,00 & $0,47^{* *}$ & 0,36 \\
\hline NPG & 9,56 & 12,11 & 0,00 & $0,62^{* *}$ & 0,37 \\
\hline $\mathrm{PP}$ & 13,52 & 13,56 & 0,00 & $0,77^{* *}$ & 0,28 \\
\hline EP & 13,9 & 6,76 & 0,01 & $0,58^{* *}$ & 0,60 \\
\hline \multicolumn{5}{|c|}{$\mathrm{R}^{2}$ / Efeito variável residual } & $0,96 / 0,21$ \\
\hline
\end{tabular}

${ }^{1}=$ classificados de acordo com o resultado do teste de médias Scott-Knott $(p<0,05)$, vide Tabela 3. M1G = massa média do grão; $N G=$ número potencial de grãos; $P P=$ potencial produtivo; $E P=$ eficiência produtiva; $R^{2}=$ coeficiente de determinação; ${ }^{* *}=$ significativo pelo teste $\mathrm{t}(p<0,01)$.

No número potencial de grãos, sob condições que foram favoráveis a maior produtividade de grãos em três safras (entressafra 2010; safra 2012/13 e; safra 2013/14 - II; Tabela 3), o coeficiente de variação genotípica foi superior ao coeficiente de variação experimental (Tabela 5), o que demonstra situação favorável para ganhos genéticos e confiabilidade nos resultados obtidos (MAPHUMULO et al., 2015). No potencial produtivo, mesmo resultado foi encontrado nas condições que foram favoráveis nas safras 2012/13 e 2013/14 - II (Tabela 5).

$\mathrm{Na}$ análise de trilha, os valores do coeficiente de determinação apresentaram superioridade, em relação aos do efeito da variável residual, em 11 das 12 análises e, ainda, maiores que 0,84 (Tabelas 4 e 5). Valores, do coeficiente de determinação, maiores que 0,90 podem ser considerados elevados e demonstram que grande parte da variação do atributo principal pode ser 
explicada/determinada pelos atributos incluídos na análise de trilha (PEREIRA et al., 2013).

A EP apresentou maior efeito direto sob a produtividade em todas as análises, com valores acima de: 0,45 (experimentos desfavoráveis) e 0,60 (experimentos favoráveis); que foram superiores aos do efeito da variável residual. $\mathrm{Na}$ mesma ordem, os coeficientes de correlação $(r)$, deste atributo com a PG, foram superiores a: 0,39 e 0,56 .

De forma semelhante, o potencial produtivo apresentou efeitos diretos maiores que o efeito da variável residual em 12 das 14 análises realizadas, enquanto que no número potencial de grãos e massa média do grão mesmo resultado foi observado em cinco e três casos, respectivamente. Resultados que demonstram que a variação dos índices de eficiência (potencial e eficiência) explicam com maior fidedignidade a variação da produtividade de grãos que a variação observada no número potencial de grãos e a massa média do grão (componentes produtivos).

Ao comparar os dois atributos agronômicos (NPG e M1G), o número potencial de grãos apresentou associação superior, com a produtividade de grãos por planta, em 10 das 12 análises realizadas $(0,26<r<0,77)$. Mesmo resultado foi observado no efeito direto (Tabelas 4 e 5), com valores entre 0,19 e 0,37 , em oito destas dez situações.

Olaniyan (2015) encontrou coeficiente de correlação superior entre a quantidade de grãos e a produtividade $(r>0,70)$ em comparação a associação do rendimento com a massa de 100 grãos $(r=0,66)$. Hernandez et al. (2014) encontraram coeficientes de 0,92 entre NG e PG e, de 0,11 entre MG e PG e Tsimba et al. (2013), coeficientes de 0,90 e 0,76 , respectivamente. Os valores encontrados por Wu et al. (2011) ficaram situados entre 0,69 e 0,78 (NG x PG) e entre 0,13 e 0,33 (M100G).

De forma semelhante, Borrás et al. (2004) e Sadras e Slafer (2012) relatam que o NPG está mais relacionado com a produtividade de grãos que a M1G. De acordo com Sadras e Slafer (2012), o número de grãos é um atributo mais plástico que a massa de grãos, enquanto que este tende de apresentar maior herdabilidade.

Neste sentido, a redução do número de grãos na espiga tem relação com estresses (hídrico, nutricional) que diminuem a taxa de assimilação líquida vegetal (KINIRY; RITCHIE, 1985) e que podem ocorrer no período vegetativo do milho (TSIMBA et al., 2013) e no início do florescimento e durante o enchimento de grãos
(TSIMBA et al., 2013; ORDÓNEZ et al., 2015). Borrás et al. (2004) relatam que umas das funções das planta no período de enchimento de grãos, em cerais, é de manter o potencial produtivo definido no estágio vegetativo de crescimento/desenvolvimento vegetal e que, o milho, em comparação ao trigo, é ineficiente em usar os assimilados antes da floração para o crescimento das sementes.

A maior associação da massa média do grão com a produtividade de grãos $(0,33<r<0,36)$ em comparação a NPG, observada em duas situações (entressafra 2010 - experimentos favoráveis e desfavoráveis), também foi observada nos trabalhos publicados por Chen et al. (2013) e Muhammad et al. (2015).

Em condições de estresse hídrico, Mansouri-Far et al. (2010) verificaram que a massa de 100 grãos foi o componente de maior importância na determinação da produtividade de grãos. Isso se relaciona com fato de que a associação dos atributos com a produtividade pode ser alterada em ambientes com algum tipo de estresse (SULTAN et al. 2014) em virtude da influência no tamanho do grão que modifica a importância dos atributos na determinação da produtividade (SADRAS; SLAFER, 2012).

\section{CONCLUSÕES}

As estimativas da variabilidade genética e da associação dos atributos, com a produtividade de grãos, sofreram influência com mudança das condições de cultivo, sendo possível identificar condições ambientais propícias a ganhos genéticos. Houve maior variabilidade genotípica nos componentes produtivos avaliados (número potencial de grãos e massa do grão), com o número potencial de grãos sendo o componente mais relacionado com a produtividade de grãos. Os índices de eficiência estimados, relacionados ao potencial produtivo e a eficiência produtiva, explicaram melhor a variação da produtividade de grãos nas condições de Cerrado Tocantinense.

\section{REFERÊNCIAS}

BAUTE, J.; HERMAN, D.; COPPENS, F.; DE BLOCK, J.; SLABBINCK, B.; DELLÁCQUA, M; PÉ, M.E.; MAERE, S.; NELISSEN, H.; INZÉ, D. Correlation analysis of the transcriptome of growing leaves with mature leaft parameters in a maize RIL population. Genome Biology, v.16, n.1, p.1-26, 2015. https://doi.org/10.1186/s13059-015-0735-9

BORRÁS, L.; SLAFER, G.A.; OTEGUI, M.E. Seed dry weight response to source-sink manipulation in 
wheat, maize and soybean: a quantitativa reappraisal. Field Crops Research, v.86, n.2, p.131146, https://doi.org/10.1016/j.fcr.2003.08.002

2004.

BU, L.; CHEN, X.; LI, S.; LIU, J.; ZHU, L.; LUO, S.; HILL, R.L.; ZHAO, Y. The effect of adapting cultivars on the water use efficiency of dryland maize (Zea mays L.) in northwestern China. Agricultura Water Management, v.148, n.1, p.1-9, 2015. https://doi.org/10.1016/i.agwat.2014.09.010

CABRAL, P.D.S.; AMARAL JÚNIOR, A.T.; FREITAS, I.L.J.; RIBEIRA, R.M.; SILVA, T.R.C. Relação causa e efeito de caracteres quantitativos sobre a capacidade de expansão do grão em milho-pipoca. Revista Ciência Agronômica, v.47, n.1, p.108-117, $2016 . \quad$ http://dx.doi.org/10.5935/1806$\underline{6690.20160013}$

CAIXETA, D.S.; FRITSHCE-NETO, R.; GRANATO, I.S.C.; OLIVEIRA, L.R.; GALVÃO, J.C.C. Early indirect selection for nitrogen use efficiency in maize. Revista Ciência Agronômica, v.46, n.2, p.369-378, $2015 . \quad$ http://dx.doi.org/10.5935/1806$\underline{6690.20150016}$

CARPICI, E.B.; CELIK, N. Determining possible relationships between yield and yield-related components in forage maize (Zea mays L.) using correlation and path analyses. Notulae Botanicae Horti Agrobotanici Cluj-Napoca, v.38, n.3, p.280285, 2010. http://dx.doi.org/10.15835/nbha3835431

CARVALHO, E.V.; AFFÉRRI, F.S.; PELUZIO, J.M.; LEÃO, F.F.; CANCELLIER, L.L.; DOTO, M.A. Eficiência e uso do nitrogênio em híbridos experimentais de milho do programa de melhoramento da Universidade Federal do Tocantins. Bioscience Journal, v.27, n.3, p.392-403, 2011.

CARVALHO, E.V.; AFFÉRRI, F.S.; PELUZIO, J.M.; DOTTO, M.A.; CANCELLIER, L.L. Nitrogen use efficiency in corn (Zea mays L) genotypes under different conditions of nitrogen and seeding date, Maydica, v.52, n.1, p.43-48, 2012.

CARVALHO, E.V; AFFÉRRI, F.S.; PELUZIO, J.M.; SANTOS, W.F.; BICUDO, S.J. Adaptabilidade na produção de massa verde e grãos de genótipos de milho no Tocantins. Revista Ciência Agronômica, v.45, n.4, p.856-862, 2014. http://dx.doi.org/10.1590/S1806$\underline{66902014000400025}$
CARVALHO, E.V.; AFFÉRRI, F.S.; PELUZIO, J.M.; LEAL. T.C.A.B.; ROTILI, E.A.; REIS, A.L.L.E. Nitrogênio em cobertura no estudo de dois grupos de genótipos de milho em Gurupi-TO. Scientia Agraria Paranaensis, v.15, n.3, p.358-364, 2016. http://dx.doi.org/10.18188/19831471/sap.v15n3p358-364

CARVALHO, E.V.; AFFÉRRI, F.S.; PELUZIO, J.M.; LEAL, T.C.A.B.; SOUZA, C.M.; NAOE, L.K. Doses de nitrogênio em cobertura na avaliação de genótipos de milho em semeadura tardia. Scientia Agraria Paranaensis, v.16, n.3, p.296-301, 2017. http://dx.doi.org/10.18188/1983-

1471/sap.v16n3p296-301

CHEN, X.; CHEN, F.; CHEN, Y.; GAO, Q.; LANG, X.; YUAN, L.; ZHANG, F.; MI, G. Modern maize hybrids in Northeast China exhibit increased yield potential and resource use efficiency desptie adverse climate change. Global Change Biology, v.19, n.3, p.923-936, 2013. http://dx.doi.org/10.1111/gcb.12093

CHEN, Y.; XIAO, C.; WU, D.; XIA, T.; CHEN, Q.; CHEN, F.; YUAN, L.; MI, G. Effects of nitrogen application rate on grain yield and grain nitrogen concentration in two maize hybrids with contrasting nitrogen remobilization efficiency. European Journal of Agronomy, v.62, n.1, p.79-89, 2015. https://doi.org/10.1016/j.eja.2014.09.008

DOVALE, J.C.; MAIA, C.; FRITSCHE-NETO, R.; MIRANDA, G.V.; CAVATTE, P.C. Genetic responses of tratis relationship to components of nitrogen and phosphorus use efficiency in maize. Acta Scientiarum. Agronomy, v.35, n.1, p.31-38, 2013. http://dx.doi.org/10.4025/actasciagron.v35i1.1523 $\underline{7}$

ECHARTE, L.; LUQUE, S.; ANDRADE, F.H.; SADRAS, V.O.; CIRILO, A.; OTEGUI, M.E.; VEJA, C.R.C. Response of maize kernel number to plant density in Argentinean hybrids released between 1965 and 1993. Field Crops Research, v.68, n.1, p.1-8, 2000. https://doi.org/10.1016/S0378-4290(00)00101-5

FILIPOVIC, M.; BABIC, M.; DELIC, N.; BEKAVAC, G.; $B A B I C, V$. Determination relevant breeding criteria by the path and factor analysis in maize. Genetika, v.46, n.1, p.49-58, 2014.

HERNÁNDEZ, F.; AMELONG, A.; BORRÁS, L. Genotypic differences among Argentinean maize 
hybrids in yield response to stand density. Agronomy Journal, v.106, n.6, p.2316-2324, 2014. http://dx.doi.org/10.2134/agronj14.0183

KINIRY, J.R.; RITCHIE, J.T. Shade-sensitive interval of kernel number of maize. Agronomy Journal, v.77, n.5, p.711-715, 1985. http://dx.doi.org/10.2134/agronj1985.000219620 $\underline{07700050012 x}$

MAKUMBI, D.; DIALLO, A.; KANAMPIU, F.; MUGO, S.; KARAYA, $H$. Agronomic performance and Genotype $x$ Environment interaction of herbicideresistant maize varieties in Eastern Africa. Crop Science, v.55, n.2, p.540-555, 2015. http://dx.doi.org/10.2135/cropsci2014.08.0593

MAPHUMULO, S.G.; DERERA, J.; QWABE, F.; FATO, P.; GASURA, E.; MAFONGOYA, P. Heritability and genetic gain for grain yield and path coefficient analysis of some agronomic traits in earlymaturing maize hybrids. Euphytica, v.206, n.1, p.225-244, 2015. https://doi.org/10.1007/s10681015-1505-1

MANSOURI-FAR, C.; SANAVY, S.A.M.M.; SABERALI, S.F. Maize yield response to deficit irragation during low-sensitive growth stages and nitrogen rate under semi-arid climatic conditions. Agricultural Water Manegement, v.97, n.1, p.1222, 2010.

https://doi.org/10.1016/j.agwat.2009.08.003

MUHAMMAD, R.W.; QAYYUM, A.; HAMZA, A.; AHMAD, M.Q.; NASEER, N.S.; LIAQAT, S.; AHMAD, B.; MALIK, W.; NOOR, E. Analysis of genetic tratis for drought tolerance in maize. Genetic and Molecular Research, v.14, n.4, p.13545-13565, 2015.

http://dx.doi.org/10.4238/2015.October.28.15

OLANIYAN, A.B. Evaluation of nitrogen fertilizer rates on growth and yield of commercially grown maize genotypes in southwestern Nigeria. Journal of Food, Agriculture \& Environment, v.13, n.2, p.122-129, 2015.

ORDÓÑEZ, R.A.; SAVIN, R.; COSSANI, C.M.; SLAFER, G.A. Yield response to heat stress as affected by nitrogen availability in maize. Field Crops Research, v.183, n.1, p.184-203, 2015. https://doi.org/10.1016/i.fcr.2015.07.010

O'NEIL, P.M.; SHANAHAN, J.F.; SCHEPERS, J.S. Use of chlorophyll fluorescence assessments to differentiate corn hydrid response to variabel water conditions. Crop Science, v.46, n.2, p.681687, 2006. http://dx.doi.org/10.2135/cropsci2005.06-0170

PEREIRA, F.B.; DOVALE, J.C.; CARNEIRO, P.C.S.; FRITSCHE-NETO, R. Relação entre os caracteres determinantes das eficiências no uso de nitrogênio e fósforo em milho. Revista Ceres, v.60, n.5, p.636645 , 2013. http://dx.doi.org/10.1590/s0034737X2013000500006

R CORE TEAM. R: A language and environment for statistical computing. R Foundation for Statistical Computing. Vienna: Austria. 2015. Disponível em: <http://www.R-project.org/>.

SADRAS, V.O.; SLAFER, G.A. Environmental modulation of yield componentes in cereals: Heritabilities reveal a hierarchy of phenotypic plasticities. Field Crops Research, v.127, n.1, p.215-224, 2012. http://dx.doi.org/0.1016/j.fcr.2011.11.014

SSERUMAGA, J.P.; OIKEH, S.O.; MUGO, S.; ASEA, G.; OTIM, M.; BEYENE, Y.; ABALO, G.; KIKAFUNDA, J. Genotype by environment interactions and agronomic performance of doubled haploids testcross maize (Zea mays L.) hybrids. Euphytica, v.207, n.2, p.353-365, 2016. http://dx.doi.org/10.1007/s10681-015-1549-2

SULTAN, M.S.; ABDEL-MONAEM, M.A.; HAFFEZ, S.H. Phenotypic and genotypic correlations, heritability and expected gains from selection for some traits of maize under two plant densities conditions. Asian Journal of Crop Science, v.6, n.1, p.49-57, 2014. http://dx.doi.org/10.3923/ajcs.2014.49.57

TOKATLIDIS, I.S.; DORDA, C.; PAPATHANASIOU, F.; PAPADOPOULOS, I.; PANKOU, C.; GEKAS, F.; NINOU, E.; MYLONAS, I.; TZANTARMAS, C.; PETREVSKA, J-K.; KARGIOTIDOU, A.; SISTANIS, I.; LITHOURGIDIS, A. Improved plant yield efficiency is essential for maize rainfed production. Agronomy Journal, v.107, n.3, p.1011-1018, 2015. http://dx.doi.org/10.2134/agronj14.0599

TSIMBA, R.; EDMEADES, G.O.; MILLNER, J.P.; KEMP, P.D. The effect of planting date on maize grain yields and yield components. Fields Crops Research, v.150, n.1, p.135-144, 2013. https://doi.org/10.1016/i.fcr.2013.05.028 
WU, Y.; LIU, W.; LI, X.; LI, M.; ZHANG, D.; HAO, Z.; WENG, J.; XU, Y.; BAI, L.; ZHANG, S.; XIE, C. LoWnitrogen stress tolerance and nitrogen agronomic efficiency among maize inbreds: comparison of multiple indices and evaluation of genetic variation. Euphytica, v.180, n.2, p.281-290, 2011. http://dx.doi.org/:10.1007/s10681-011-0409-y ZWART, S.J.; BASTIAANSSEN, W.G.M. Review of measured crop water productivity values for irrigated wheat, rice, cotton and maize. Agricultural Water Management, v.69, n.2, p.115133, 2004.

https://doi.org/10.1016/j.agwat.2004.04.007

Recebido para publicação em 19/11/2017

Revisado em 17/05/2018

Aceito em 29/06/2018 